\title{
EMG Analysis of Dominant and Non-Dominant Arm of Latissimus Dorsi Muscles in Bowlers of Karad, Maharashtra, India
}

\author{
Pradip Raghunath Lad ${ }^{1}$, Dhairysheel Sanjay Patil ${ }^{2}$, Smita Chandrakant Patil ${ }^{3}$, Khushboo Trishant Chotai ${ }^{4}$ \\ 1, 2 Krishna College of Physiotherapy, Krishna Institute of Medical Sciences," Deemed to Be University”, Karad, \\ Maharashtra, India. 3, 4 Department of Physiotherapy, Krishna Institute of Medical Sciences, "Deemed to be \\ University", Karad, Maharashtra, India.
}

\section{ABSTRACT}

\section{BACKGROUND}

Latissimus dorsi plays an important role in bowlers. Different bowling techniques recruit latissimus dorsi at different phases of bowling. This causes the muscle to undergo excessive contraction than its limit. Despite widespread knowledge about the recruitment of latissimus dorsi in bowling, there is limited electromyographic (EMG) studies about latissimus dorsi. It is necessary to evaluate and document the recruitment of latissimus dorsi in dominant and non-dominant arm in bowlers, which is focused in this study. This study was done to analyse the surface EMG activity of latissimus dorsi muscle in dominant and non-dominant arm during bowling.

\section{METHODS}

A cross-sectional analytical study was carried out among 96 professional cricket bowlers from deluxe cricket club and Yashwanthrao Chavan's cricket club in Karad. EMG electrodes were applied over the latissimus dorsi muscle and the bowlers were asked to perform the action of bowling. The root mean square (RMS) and mean peak amplitude of the dominant and non-dominant arm were recorded.

\section{RESULTS}

The RMS $(12.45 \pm 2.84)$ values and mean peak amplitude $(131.77 \pm 44.11)$ of the dominant latissimus dorsi varied significantly from non-dominant muscle $(\mathrm{P}=$ 0.0015). Wilcoxon test was utilized for analysis of within group parameters.

\section{CONCLUSIONS}

The result of the present study showed strong evidence to support high recruitment of dominant latissimus dorsi in professional bowlers suggests the changes occurred in the physiological as well as biomechanical muscular structure.
Corresponding Author: Dr. Smita Chandrakant Patil, Assistant Professor, Krishna Institute of Medical Sciences, "Deemed to be University", Karad, Maharashtra, India.

E-mail: smitakanase@gmail.com

DOI: $10.14260 /$ jemds/2021/630

How to Cite This Article:

Lad PR, Patil DS, Patil SC, et al. EMG analysis of dominant and non-dominant arm of latissimus dorsi muscles in bowlers of Karad, Maharashtra, India. J Evolution Med Dent Sci 2021;10(36):3088-3093, DOI: $10.14260 /$ jemds/2021/630

Submission 25-03-2021,

Peer Review 29-05-2021,

Acceptance 05-06-2021,

Published 06-09-2021.

Copyright (C) 2021 Pradip Raghunath Lad et al. This is an open access article distributed under Creative Commons Attribution License [Attribution 4.0 International (CC $B Y 4.0)]$

\section{KEY WORDS}

EMG, Dominant, Non-Dominant, Latissimus Dorsi, Fast Bowlers 


\section{BACKGROUND}

Cricket is considered as one of the oldest played sport in the world. It is the second most popular sport worldwide. Cricket involves combined movement of the entire upper limb and owing to the aggressive nature of the sport, its prone to cause injuries of the shoulder and wrist complex. There are 3 skills in cricket - batting, bowling and fielding. These skills require aggressive and combined mechanics of upper limb which can lead to injuries. Fast bowling, especially, can cause non-contact injuries of the shoulder and wrist. Acceleration of the upper limb occurs in a sequence of proximal to distal joint rotations followed by muscle contractions in the same sequence. The understanding of the biomechanics of joints of upper extremity helps to determine the mechanism of these injuries. ${ }^{1}$ Bowling consists of following phases -1 . Run-up to the release while holding the ball, 2 . Leap into the pre-delivery stride, 3. Mid-bound, 4. Back foot contact, 5. Front foot contact, 6. Ball release and 7 . Follow through. ${ }^{2}$ Fast bowling consists of concentric contraction of the internal rotators of the shoulders during acceleration counteracted by eccentric contraction of the external rotators during deceleration phase. Due to the high physical demands of cricket, overuse injuries and injuries due to improper mechanics are commonly seen in fast bowlers. It has been proposed that EMG is a basic non-invasive technique used for the quantification of muscle activity during movement. ${ }^{1}$ EMG has proven to be an essential tool in biomechanical investigations and is used to identify the exact muscle function, injury location, pain, fatigue and other abnormalities. For adequate sports performance, proper body mechanics and proper technique is essential. ${ }^{2}$ The most commonly activated skeletal muscles during fast bowling are triceps brachii, deltoid, pectoralis major, latissimus dorsi, trapezius, infraspinatus, supraspinatus and serratus anterior. Due to excessive stress and high physical demands placed on these muscles during the bowling action, these muscles are more commonly subjected to tendinitis, strain, fatigue, acute injury and rupture. ${ }^{3}$ In cricket bowlers, overuse injuries occur most commonly during ball delivery which involves repetitive twisting, extension, contraction and rotation of the upper limb. According to literature, the EMG studies on upper limb muscles has not fully been explored. ${ }^{4}$ Hence this study has been undertaken wherein the focus will be on latissimus dorsi muscle of both the dominant and non-dominant arms. This will not only create awareness about the improper mechanics but will also shed light on repetitive and overuse injuries and will help explore techniques which will help avoid such injuries in bowlers.

\section{Objectives}

- To analyze the RMS value attained during bowling. Three trials of EMG signal per bowler will be calculated from dominant and non-dominant arm of latissimus dorsi muscles.

- To analyse the mean of peak amplitude of the normalized EMG signals of both dominant and non-dominant arm of latissimus dorsi muscles during bowling.

- To compare the final scores of peak amplitude and RMS values with dominant and non-dominant arm of latissimus dorsi muscles.

\section{METHODS}

An analytical cross-sectional study was carried out among 96 professional (formula below) cricket fast bowlers at Krishna College of Physiotherapy, Karad, Maharashtra from November 2019 to April 2020. The participants were selected by simple random sampling method based on the inclusion and exclusion criteria. The bowlers selected were from deluxe cricket club and Yashwanthrao Chavan's cricket club of Karad Ethical clearance was obtained from the Institutional Ethical Committee of Krishna Institute of Medical Sciences, Karad. Inclusion criteria of the study was male bowlers between the age of 18 - 25 years having no history of injury and who were willing to participate. Exclusion criteria of the study was players who had skin allergies, neurological problems, etc. Spin bowlers and slow-paced bowlers were also excluded from this study. For the purpose of statistical analysis, the participants were divided into two groups.

Group I - the participants were allotted as per their age,
A. $18-19$ years
B. $20-21$ years
C. 22 - 23 years
D. $24-25$ years

Group II - the participants were allotted as per their experience for bowling

A. Less than and 2 years of experience

B. 2 - 3 years of experience

C. More than 3 years of experience.

\section{Sample Size $=96$}

Formula for sample size

$N=p(q) / L^{2}=p(100-p) / L^{2}=41(100-41) / 5^{2}=96$

Outcome measures - EMG studies were carried out first in group I and comparative analysis was done between the age groups. After this, the same study was done on group II and comparative analysis was done between the years of experience of bowling. Root mean square value and peak amplitude values of the latissimus dorsi of the dominant and non-dominant arm were recorded when the participant was asked to perform the bowling.

\section{Statistical Analysis}

Statistical analysis for present study was done manually as well as using the statistics software INSTAT so as to verify the results obtained. Various statistical measures such as mean, standard deviation (SD) and paired and unpaired test of significance were utilized for this purpose. Probability values less than 0.05 were considered statistically significant and probability values less than 0.0001 were considered statistically extremely significant.

\section{RESULTS}

Based on the data accumulated and analyzed, the study was divided into 2 parts, one as per the age of the bowler and the other as per the years of experience of bowling. The findings were summarized in a tabular and graph format. For the first 
part of the study, the participants were divided into 4 groups as per their age.

1. 18 - 19 years

2. $20-21$ years

3. $22-23$ years

4. $24-25$ years

The relation between the root mean square value of latissimus dorsi and the age of the bowler can be represented by the graph. As per the statistical representation, it can be seen that the mean value of root mean square of dominant arm in the age group 18 - 19 years was 12.45 whereas for the nondominant arm it was 8.12. Similarly, in the age group $20-21$ years, it can be seen that the mean root mean square value in dominant arm was 13 whereas in non-dominant arm it was 8.2. The mean root mean square value of the age group 22 - 23 years was 14.58 of dominant arm whereas it was 9.29 for the non-dominant arm. In the age group of $24-25$ years, it can be seen that, the average value of root mean square was 15.83 for the dominant arm and 9 for the non-dominant arm. The statistics represent that as the age progresses, the root mean square value of the dominant arm progresses. It also represents that the root mean square value of the dominant arm i.e. The arm used during bowling is higher as compared to that of non-dominant arm. The relation between the peak amplitude value of latissimus dorsi and the age of the bowler can be represented by the graph.

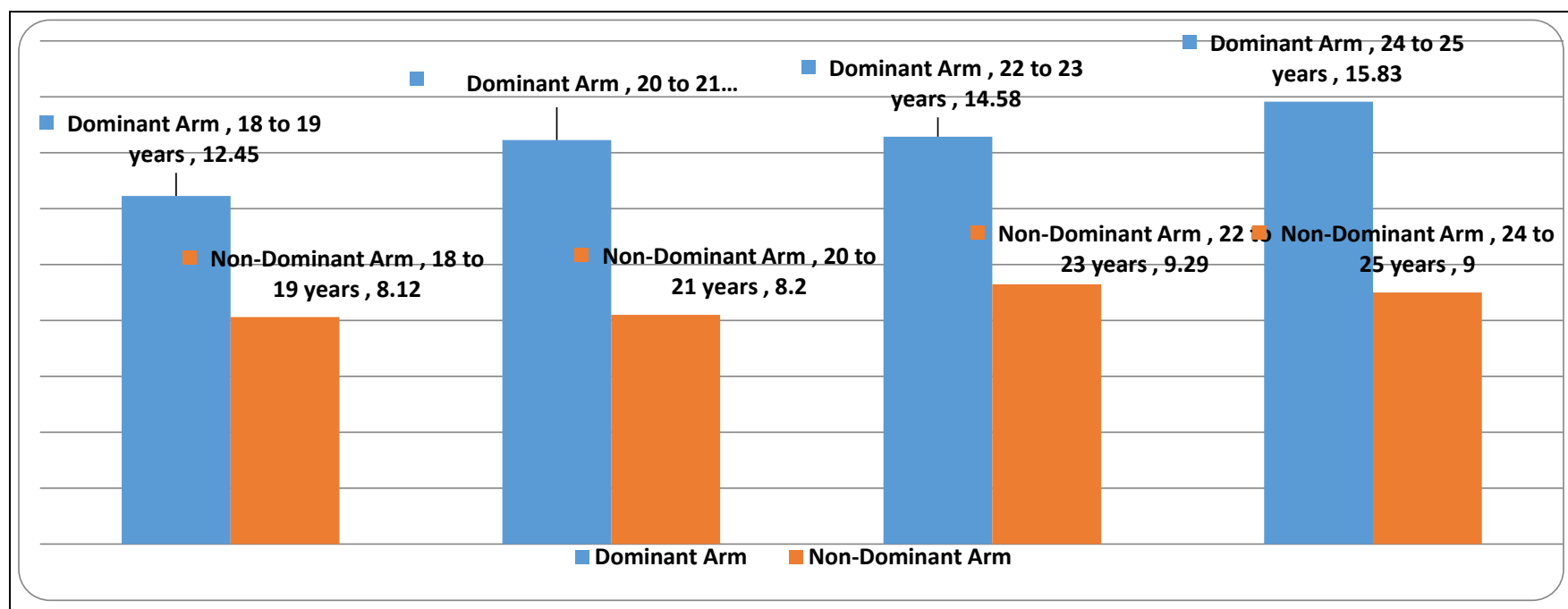

Figure 1. Relation between the Root Mean Square Value of Latissimus Dorsi and the Age of the Bowler

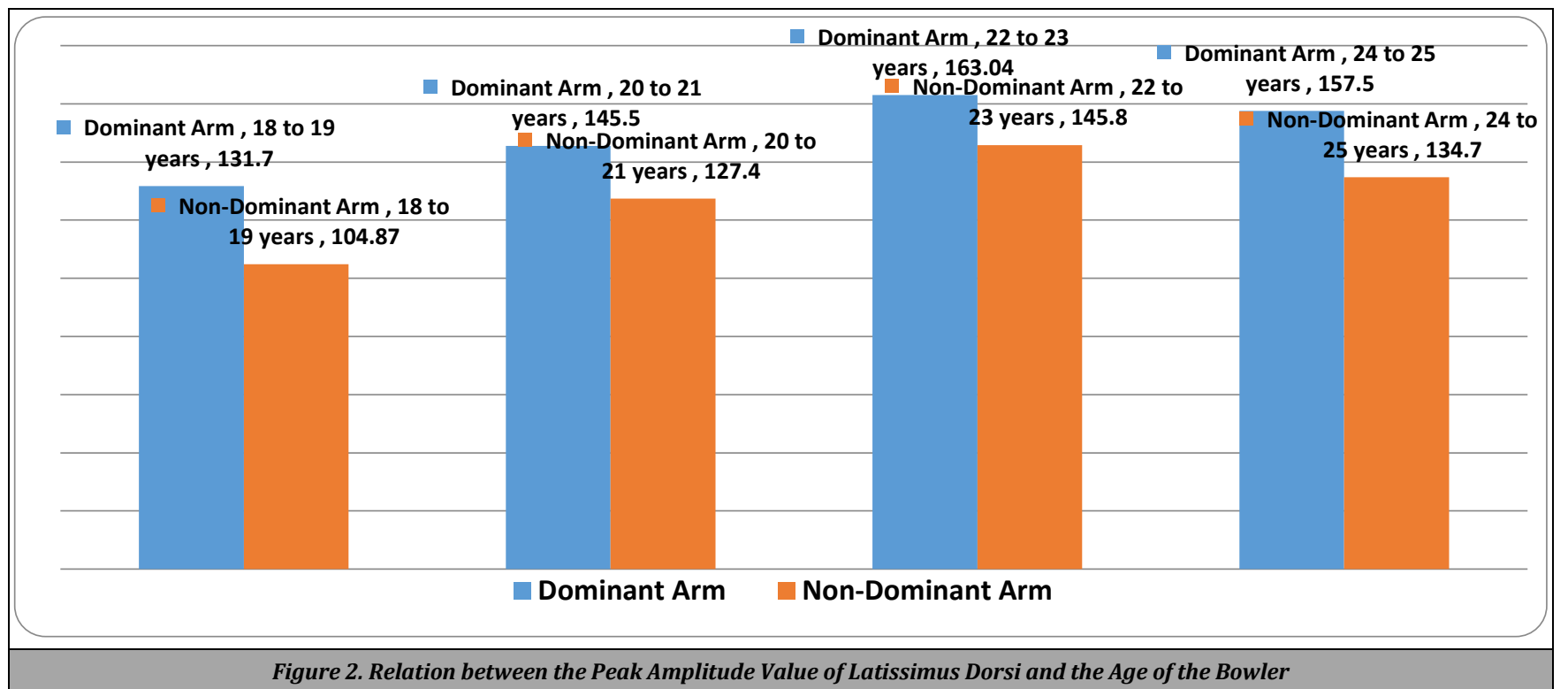

As per the statistical representation, it can be seen that the mean peak amplitude value of the dominant arm in the age group 18 - 19 years was 131.7 whereas for the non-dominant arm it is 104.87. Similarly, in the age group of $20-21$ years, it can be seen that the mean peak amplitude value in dominant arm was 145.5 whereas in non-dominant arm was 127.4. The mean peak amplitude value of the age group 22 - 23 years was 163.04 of dominant arm whereas it was 145.8 for the nondominant arm. In the age group of 24-25 years, it can be seen that, the mean peak amplitude value was 157.5 for the dominant arm and 134.7 for the non-dominant arm. It also represents that the mean peak amplitude value of the dominant arm i.e. the arm used during bowling is higher as compared to that of non-dominant arm. For the second part of the study, the participants were divided into 3 groups as per their years of experience in bowling - , Less than 2 years of experience, 2 - 3 years, More than 3 years. 


\begin{tabular}{|c|c|c|c|c|c|c|c|}
\hline Age Group & & $\mathbf{N}$ & Minimum Value & Maximum Value & Mean & S. D & P Value \\
\hline \multirow{4}{*}{18 - 19 years. } & RMS value of dominant arm & 24 & 8 & 17 & 12.45 & 2.84 & \multirow{4}{*}{$\begin{array}{c}P=0.0015 \text { (significant) } \\
P=0.1532 \text { (non-significant) }\end{array}$} \\
\hline & RMS value of non- dominant arm & 24 & 7 & 10 & 8.12 & 1.96 & \\
\hline & Peak amplitude value of dominant arm & 24 & 54 & 233 & 131.77 & 44.11 & \\
\hline & Peak amplitude of non-dominant arm & 24 & 25 & 210 & 104.87 & 79.30 & \\
\hline \multirow{4}{*}{$19-20$ years } & RMS value of dominant arm & 24 & 8 & 17 & 12.45 & 2.84 & \multirow{4}{*}{$\begin{array}{c}P=0.0015 \text { (significant) } \\
P=0.1532 \text { (non-significant) }\end{array}$} \\
\hline & RMS value of non-dominant arm & 24 & 7 & 10 & 8.12 & 1.96 & \\
\hline & Peak amplitude value of dominant arm & 24 & 54 & 233 & 131.77 & 44.11 & \\
\hline & Peak amplitude of non-dominant arm & 24 & 25 & 210 & 104.87 & 79.30 & \\
\hline \multirow{4}{*}{$20-21$ years } & RMS value of dominant arm & 24 & 7 & 16 & 14.45 & 2.67 & \multirow{4}{*}{$\begin{array}{c}\mathrm{P}<0.0001 \text { (significant) } \\
\mathrm{P}=0.0629 \text { (non-significant) }\end{array}$} \\
\hline & RMS value of non-dominant arm & 24 & 4 & 12 & 8.20 & 1.44 & \\
\hline & Peak amplitude value of dominant arm & 24 & 106 & 223 & 145.5 & 34.3 & \\
\hline & Peak amplitude value of non-dominant arm & 24 & 74 & 189 & 127.4 & 31.09 & \\
\hline \multirow{4}{*}{$22-23$ years } & RMS value of dominant arm & 24 & 8 & 18 & 14.58 & 2.79 & \multirow{4}{*}{$\begin{array}{c}\mathrm{P}<0.0001 \text { (significant) } \\
\mathrm{P}=0.0647 \text { (non-significant) }\end{array}$} \\
\hline & RMS value of non-dominant arm & 24 & 6 & 15 & 9.29 & 2.23 & \\
\hline & Peak amplitude of dominant arm & 24 & 104 & 244 & 163.04 & 32.55 & \\
\hline & Peak amplitude of non-dominant arm & 24 & 91 & 210 & 145.8 & 30.41 & \\
\hline \multirow{4}{*}{$24-25$ years } & RMS value of dominant arm & 24 & 8 & 17 & 15.83 & 3.62 & \multirow{4}{*}{$\begin{array}{c}P<0.0001 \text { (significant) } \\
P=0.01583 \text { (non-significant) }\end{array}$} \\
\hline & RMS value of non-dominant arm & 24 & 6 & 14 & 9.45 & 2.20 & \\
\hline & Peak amplitude of dominant arm & 24 & 96 & 255 & 157.5 & 51.2 & \\
\hline & Peak amplitude of non-dominant arm & 24 & 67 & 216 & 134.7 & 58.2 & \\
\hline
\end{tabular}
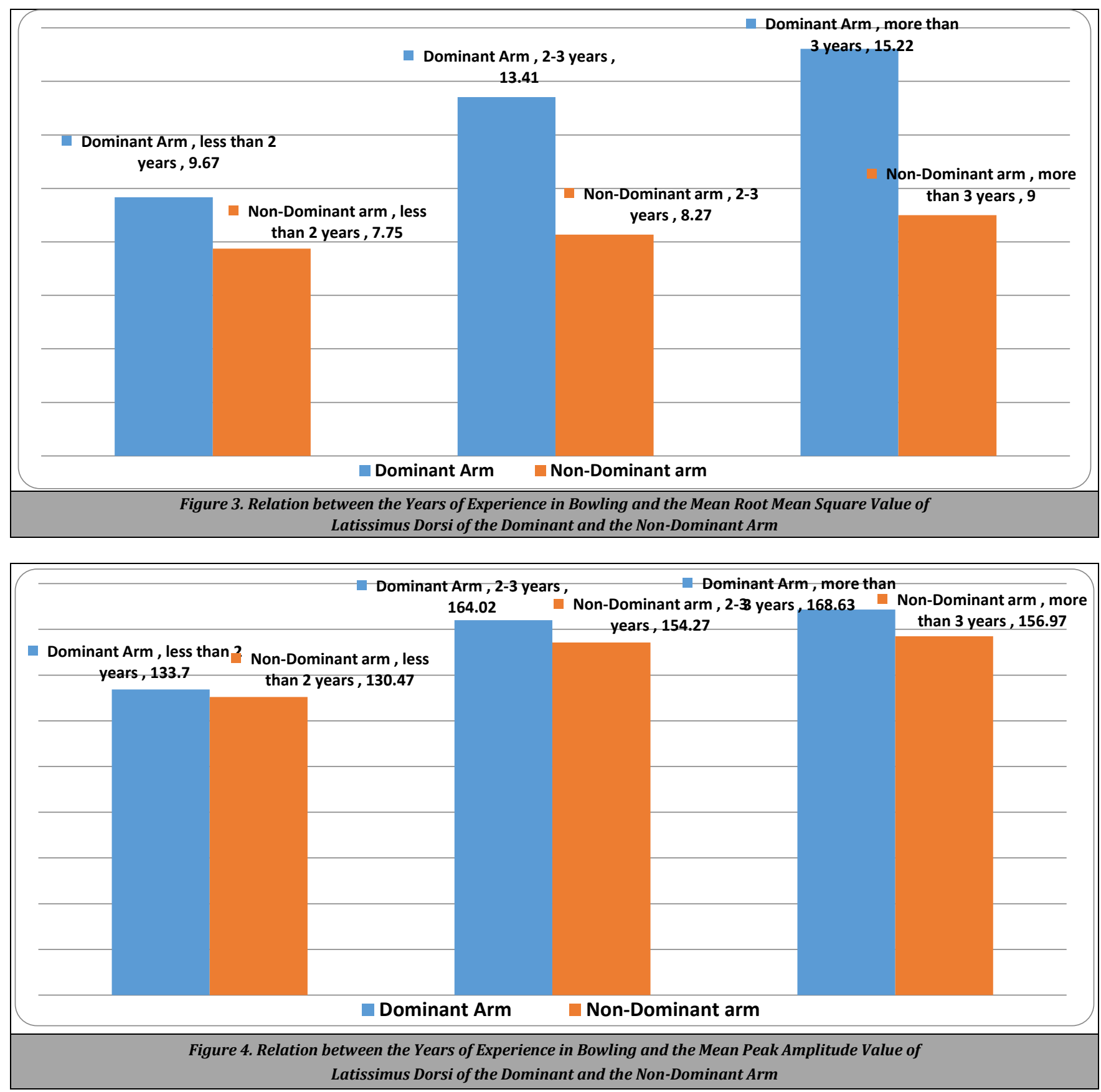

The statistical analysis represents the relation between the years of experience in bowling and the mean root mean square value of latissimus dorsi of the dominant and the non- dominant arm. In participants who had less than 2 years of experience in bowling, the mean root mean square value for the dominant arm was 9.67 whereas for the non-dominant 
arm was 7.75. The mean root mean square value for the dominant arm in participants who had 2 - 3 years of experience in bowling was 13.41 whereas for the nondominant arm the mean value was 8.27. The mean root mean square value in participants who had more than 3 years of experience had the values of 15.22 for dominant arm and 9.25 for the non-dominant arm. The statistics represent that as the experience in bowling increases, the value of root mean square for the dominant arm also increases.

The statistical analysis represents the relation between the years of experience in bowling and the mean peak amplitude value of latissimus dorsi of the dominant and the non- dominant arm. In participants who had less than 2 years of experience in bowling, the mean peak amplitude value for the dominant arm was 133.7 whereas for the non-dominant arm it was 130.47. The mean peak amplitude value for the dominant arm in participants who had 2 - 3 years of experience in bowling was 164.02 whereas for the nondominant arm the mean value was 154.27 . The mean peak amplitude value in participants who had more than 3 years' experience had the values of 168.83 for dominant arm and 156.97 for the non-dominant arm. The statistics represent that as the experience in bowling increases, the value of peak amplitude for the dominant arm also increases.

\begin{tabular}{|c|c|c|c|c|c|c|c|}
\hline Bowling Experience & & $\mathbf{N}$ & Minimum Value & Maximum Value & Mean & S. D & P Value \\
\hline \multirow{4}{*}{$\begin{array}{c}\text { Participants with less than } 2 \text { years of } \\
\text { experience in bowling }\end{array}$} & RMS value of dominant arm & 12 & 7 & 14 & 9.67 & 2.01 & \multirow{4}{*}{$\begin{array}{l}P=0.0110 \\
P=0.0287\end{array}$} \\
\hline & RMS value of non-dominant arm & 12 & 5 & 13 & 7.75 & 1.28 & \\
\hline & Peak amplitude of the dominant arm & 12 & 54 & 233 & 133.7 & 51.92 & \\
\hline & Peak amplitude of non-dominant arm & 12 & 25 & 211 & 69.4 & 98.19 & \\
\hline \multirow{4}{*}{$\begin{array}{l}\text { Participants with } 2-3 \text { years of } \\
\text { experience in bowling }\end{array}$} & RMS value of dominant arm & 48 & 6 & 17 & 13.41 & 2.87 & \multirow{4}{*}{$\begin{array}{l}P<0.0001 \\
P=0.0318\end{array}$} \\
\hline & RMS value of non-dominant arm & 48 & 6 & 13 & 8.27 & 1.63 & \\
\hline & Peak amplitude of dominant arm & 48 & 120 & 228 & 164.02 & 25.94 & \\
\hline & Peak amplitude of non-dominant arm & 48 & 107 & 219 & 154.27 & 24.94 & \\
\hline \multirow{4}{*}{$\begin{array}{l}\text { Participants with more than } 3 \text { years } \\
\text { of experience in bowling }\end{array}$} & RMS value of dominant arm & 24 & 9 & 18 & 15.22 & 3.58 & \multirow{4}{*}{$\begin{array}{l}\mathrm{P}<0.0001 \\
\mathrm{P}=0.0252\end{array}$} \\
\hline & RMS value of non-dominant arm & 24 & 7 & 15 & 9.25 & 2.11 & \\
\hline & Peak amplitude of dominant arm & 24 & 104 & 244 & 168.63 & 40.20 & \\
\hline & Peak amplitude of non-dominant arm & 24 & 94 & 229 & 156.97 & 39.18 & \\
\hline \multicolumn{8}{|c|}{ Table 2. Participants as Per Their Bowling Experience } \\
\hline
\end{tabular}

\section{DISCUSSION}

The study was conducted to analyse and compare the root mean square value and peak amplitude of the latissimus dorsi muscle in dominant and non-dominant arm in professional fast cricket bowlers. EMG was used for this study as it provides a quantitative measure to study the firing of muscle fibers of the dominant and non-dominant arm during the phases of bowling. The study was conducted in 2 parts. For the first part, the participants were divided into 4 groups as per their age. The results of the first part of the study signify that as the age increases, the root mean square value also increases linearly. It is also seen that the root mean square value of the dominant arm is higher than that of the non-dominant arm. This could be due to excessive recruitment of the fibers of latissimus dorsi muscle of dominant hand during the phases of bowling as compared to the non-dominant hand. The results also signify that participants who belonged to the younger age group of 18 - 19 years had a significant lower RMS value as compared to those who belonged to the age group of $24-25$ years. The $P$ value signifies the relationship between the RMS value and the age group. The peak amplitude value for the first group in the dominant and non-dominant arm did not have any significant difference. For the second part of the study, the participants were divided into 3 groups as per their experience in bowling. It was seen that as the experience in bowling increases, the root mean square value of the dominant as well as the nondominant arm increases. The peak amplitude values did not show any significant variation as compared to root mean square value. The dominant arm values being higher as compared to the non-dominant arm. In a study conducted on the EMG analysis of the shoulder and wrist muscles of the semi-fast bowlers during yorker and bouncer delivery, it was seen that the RMS value of latissimus dorsi muscle was greater in yorker delivery than in the bouncer delivery. ${ }^{1}$ The study also showed that during the yorker delivery, the contraction of supraspinatus was lesser as compared to latissimus dorsi muscle and infraspinatus muscle ${ }^{1}$ This causes an imbalance between the agonist and antagonistic group which could be a risk factor for shoulder injuries. ${ }^{1}$ Literature suggests that not many studies have been conducted on determining the root mean square values of muscles employed during the action of bowling.2. A study was conducted to determine the significance of electromyographic analysis on upper limb muscles of cricket bowlers, which suggested that there is a higher incidence of shoulder and wrist injuries in professional fast bowlers as compared to spin / slow bowlers. ${ }^{2}$ This could be due to excessive forceful demands placed on the muscles during the phases of bowling in fast bowling. It is necessary to study the recruitment and effects of excessive demands placed on the muscles during bowling, to create awareness about the proper mechanics and prevent injuries. ${ }^{2}$ A similar study was conducted to determine the EMG signals of 3 heads of triceps brachi. The study showed that during different phases of bowling, levels of eccentric contraction of the triceps brachi occur. ${ }^{3}$ The study also indicates that during different phases of bowling, different muscles are recruited, improper technique of bowling can put excessive stresses on these muscles which in turn could lead to injuries. ${ }^{3}$ Latissimus dorsi injuries in cricketers is an understudied topic. Literature suggests that these injuries have come into light only recently. ${ }^{4}$

The key component which leads to injury of latissimus dorsi muscle is forced internal rotation at full extension. This inadvertently places the latissimus dorsi muscle under eccentric loading. Improper biomechanics or inadequate technique can put excessive load on the muscle which in turn could lead to injuries. ${ }^{4}$ Awareness of the injuries of latissimus dorsi can help create awareness about suspecting a case of latissimus dorsi injury when a bowler complaints of posterior axillary pain. ${ }^{4}$ In a study conducted on muscle activity in throwing with dominant and non-dominant arm, it was reported that there was no significant variation in the values of peak amplitudes obtained during the phase of throwing in dominant and non-dominant arm. ${ }^{5}$ Further research on the variations seen in different muscle groups of the same limb can be done. However, the results of this study show that there was 
no significant variation in the peak amplitude values of latissimus dorsi muscle of the dominant and non-dominant arm.

This study shed light on how the latissimus dorsi muscle is employed during fast bowling in cricketers. It also provides comparative analysis with EMG interpretation between the dominant and non-dominant latissimus dorsi muscle of arm.

\section{CONCLUSIONS}

The findings conclude that the root mean square value of latissimus dorsi muscle increases as the experience of bowling increases as well as the age of the bowler increases. However, there were no significant variation noted in peak amplitude values of the dominant and non-dominant arm. The study also provides an understanding that more muscle fibres of the dominant arm are recruited during bowling when compared to non-dominant.

Data sharing statement provided by the authors is available with the full text of this article at jemds.com.

Financial or other competing interests: None.

Disclosure forms provided by the authors are available with the full text of this article at jemds.com.

\section{REFERENCES}

[1] Hazari A, Warsi M, Agouris I. Electromyography analysis of shoulder and wrist muscles in semi professional cricket fast bowlers during bouncer and yorker delivery. A cross sectional comparative study. International Journal of Physicaal Education, Sports and Health 2016;3(6):77-87.

[2] Ahamed NU, Sundaraj K, Ahmad B, et al. Significane of the electromyographic analysis of the upper limb muscles of cricket bowlers; recommendations from studies of overhead-throwing athletes. Journal of Mechanics in Medicine and Biology 2014;14(4):1430005.

[3] Ali MA, Sundaraj K, Ahmad RB, et al. Semg activities of the three heads of the triceps brachii muscle during cricket bowling. Journal of Mechanics in Medicine and Biology 2016;16(5):1650075.

[4] Naidu KS, James T, Rolstein AH, et al. Latissimus dorsi and teres major avulsions in cricketers: a case series and literature review. Clinical Journal of Sport Medicine 2017;27(3):e24-8.

[5] Kuhtz-Buschbeck JP, Keller P. Muscle activity in throwing with the dominant and non-dominant arm. Cogent Medicine 2019;6(1):1678221. 